\title{
Carcinoma of Unknown Primary Site: Outcomes in Patients with a Colorectal Molecular Profile Treated with Site Specific Chemotherapy*
}

\author{
F. Anthony Greco ${ }^{1,2}$, Wayne J. Lennington ${ }^{3}$, David R. Spigel ${ }^{1,2}$, Gauri R. Varadhachary ${ }^{4}$, \\ John D. Hainsworth ${ }^{1,2}$ \\ ${ }^{1}$ Sarah Cannon Cancer Center and Research Institute, Nashville, USA; ${ }^{2}$ Tennessee Oncology, PLLC, Nashville, USA; ${ }^{3}$ Associated \\ Pathologists, Inc., Brentwood, USA; ${ }^{4}$ University of Texas MD Anderson Cancer Center, Houston, USA. \\ Email: fgreco@tnonc.com, aso@scresearch.net
}

Received December $17^{\text {th }}, 2011$; revised January $20^{\text {th }}, 2012$; accepted February $9^{\text {th }}, 2012$

\begin{abstract}
Purpose: Accurate identification of carcinoma of unknown primary site (CUP) patients with colorectal sites of origin may improve outcomes by directing colorectal chemotherapy rather than empiric chemotherapy. Clinical features, standard pathologic evaluation, treatment response, and survival of CUP patients whose tumors had colorectal genetic signatures were examined. Patients and Methods: We prospectively tested paraffin-embedded biopsies from 213 CUP patients using mRNA-based RT-PCR assays, and identified patients with colorectal genetic signatures. Results: Assays were successful in 185 specimens (87\%); 32 (17\%) had colorectal genetic signatures. All 32 patients had carcinoma; colonoscopy was normal in 30 patients studied; 29 patients (90\%) had typical metastatic sites; 17 (53\%) had consistent IHC profiles (CK20+, CDX2+). Twenty-nine patients (90\%) received first- and/or second-line colorectal chemotherapy regimens (response rates of $69 \%$ and 54\%, respectively). Median, 2- and 4-year survivals for all 32 patients were 21 months, $42 \%$, and $35 \%$, respectively. Conclusion: The majority of CUP patients with colorectal molecular profile diagnoses responded to site-specific chemotherapy; median survival was similar to patients with known advanced colorectal carcinoma and superior to expected survival of CUP patients receiving empiric chemotherapy. Molecular profile assays can identify CUP patients with colorectal carcinoma; colorectal chemotherapy appears to improve outcomes.
\end{abstract}

Keywords: Carcinoma of Unknown Primary Site; Colorectal Cancer; Molecular Profiling

\section{Introduction}

Cancer of unknown primary site (CUP) patients are a large and heterogeneous group with clinically unidentified primary tumor sites and are ideal candidates for classification by molecular profiling [1,2]. Relatively specific gene expression profiles for classifying human tumors have been reported and several molecular profiling assays are commercially available as a diagnostic test for determining the possible tissue of origin for these patients [3-6]. Although molecular profiling assays appear to be quite accurate in predicting the primary tumor site [7-11], there is a need to determine if the outcome or prognosis of CUP patients can be improved by therapeutic decisions based on the molecular assay diagnosis.

Empiric chemotherapy regimens have been considered

"Conflict of interest statement: Dr. Greco received honoraria from bioTheranostics. All other authors have no conflict of interest to disclose. Funded in part by a grant from bioTheranostics. the standard first line approach for the majority of patients with CUP $[1,12]$. These regimens are relatively broad spectrum, usually including a platinum agent in combination with either a taxane or gemcitabine. The overall results have been modest, usually producing objective response rates of $25 \%-35 \%$ and median survivals of about 9 months. Although these empiric regimens are relatively effective for some of the occult primary sites represented in the CUP population (e.g. lung, ovary, breast), they are ineffective for patients with advanced colorectal carcinoma. Since the survival of patients with advanced colorectal cancer has improved considerably in the past 15 years $[13,14]$ (median survival from about 11 months to now 24 months), the appropriate recognition and treatment of this subset of CUP patients may be important.

The development of molecular profiling raises the important question as to whether treatment guided by the molecular assay diagnosis is superior to empiric therapy, 
particularly for the subset of patients with CUP and colorectal molecular signatures. In this report, we detail the clinicopathologic features, response to treatment, and survival of 32 CUP patients who were predicted to have colorectal cancer by molecular profiling. Profiling results were obtained during initial clinical evaluations or during the clinical course in all patients, and results were used to guide site-specific therapy in most patients.

\section{Patients and Methods}

\subsection{Patient Selection and Study Design}

This study was a collaboration of investigators from two institutions and includes patients with CUP with a molecular profile assay diagnosis of colorectal carcinoma. Patients identified at each institution were part of prospective studies primarily designed to correlate the molecular profile assay diagnosis with clinicopathologic features. All patients had tissue biopsies; fine needle aspirates were excluded. Cohort A included 42 patients evaluated between March 2004 and June 2007 at the University of Texas M D Anderson Cancer Center and previously published [7]. Eleven of these 42 biopsy specimens had a molecular profile assay (Veridex) diagnosis of colorectal carcinoma. Treatment decisions for these patients were based on clinical features, radiographic data, immunohistochemistry (IHC) and the results of the molecular profile assay as per the standard at the University of Texas, M D Anderson Cancer Center, Houston, Texas, for CUP patients.

Cohort B included 171 patients who had biopsy specimens tested at the Sarah Cannon Cancer Center, Nashville, Tennessee, between March 2008 and January 2010. Twenty-one of these 171 biopsy specimens had a molecular profile assay (bioTheranostics) diagnosis of colorectal carcinoma. The majority of patients were treated based upon the results of clinicopathologic features, immunohistochemical (IHC) staining and the results of molecular profile assay. The definition of CUP for both Cohorts A and B was the same and included no detectable primary tumor site identified after diagnostic evaluation including: complete history, physical examination, complete blood counts, chemistry profile, computed tomography scans of the chest, abdomen and pelvis, mammography in women, a serum prostate specific antigen determination in men, appropriate studies for evaluation of any specific signs or symptoms and pathologic evaluation of the biopsy specimen.

Clinical and pathological information was collected on these 32 patients (11 in Cohort A and 21 in Cohort B). Patient demographics, metastatic pattern of spread, results of pathologic evaluation (including IHC staining), chemotherapy administered, response to treatment and overall survival were obtained.

The institutional review boards of MD Anderson Cancer Center and the Sarah Cannon Research Institute approved the study which included Cohort A and B, respectively.

\subsection{Assay Procedures}

In Cohort A, formalin fixed paraffin embedded (FFPE) biopsy tissue blocks were collected and sent to Veridex where the CUP molecular assay was performed. In Cohort B, FFPE specimens were sent to bioTheranostics for the CancerTYPE ID assay. For both assay procedures specimens were coded, and those performing the assays did not have any specific clinicopathologic information, other than the biopsy location, sex of the patient and one hematologic and eosin-stained slide of the biopsy specimen.

In Cohort A, the Veridex assay was utilized. This assay is not currently commercially available and consists of ten gene markers specific for 6 cancers, including: colon, pancreas, lung, breast, prostate and ovarian primary tumors. The assay was developed selecting markers from a large number of different candidate markers, based on reverse transcriptase-polymerase chain reaction (RT-PCR) results from tumors of known origin. The assay specifics have been previously published [15].

RNA was isolated from 6 to $910 \mu \mathrm{m}$ FFPE tissue sections. A tissue section stained with hematoxylin and eosin was performed on an adjacent section to ensure that more than $10 \%$ tumor cells were present in the tissue specimen. Results were reported as probabilities for each of the six primary cancer types included in the assay. If the molecular profile did not fit any of the six tissue types, the result was reported as "other". Prediction of the tissue of origin was assigned, based on the highest probability reported.

In Cohort B, the commercially available CancerTYPE ID assay (bioTheranostics) was performed on FFPE specimens. This RT-PCR assay measures 92 genes and has been validated in 39 tumor types [3] The FFPE tissue blocks were sectioned to obtain three unstained $10 \mu \mathrm{m}$ sections and one adjacent hematoxylin and eosin-stained section to confirm the presence of tumor in target areas enriched for tumor content.

Areas of the biopsy that contained $\geq 40 \%$ tumor were isolated and marked by a pathologist. Laser microdissection was later incorporated into the method and $40 \%$ of the specimens were harvested with this procedure.

The enriched tumor had total RNA extracted from the lysed cells and reverse transcribed to generate cDNA for the assay [3]. The assay (RT-PCR) was performed on an ABI 7900HT instrument using Taq Man technology (Applied Biosystems, Foster City, CA). The expression profile for each biopsy sample was compared with a tu- 
mor gene-expression database using a proprietary algorithm to predict the most likely primary tissue of tumor origin and histologic subtype.

Results were reported as ranked probabilities with single top predictions of primary tissue of origin and histologic subtype. The algorithm reports other possibilities in rank order based on probability, reflecting overlap in similar gene-expression profiles. In all specimens the probability of intestinal or colorectal carcinoma was the highest rank probability (greater than $50 \%$ probability in all samples).

\subsection{Statistical Methods}

The assumption was made that CUP patients with a colorectal molecular profile will have similar response rates and survival as patients with known advanced colorectal carcinoma when treated with colorectal chemotherapy regimens. A one arm binomial one-sided design tested the null hypothesis that the true objective response rate is $30 \%$ (range $25 \%-35 \%$ ) for CUP patients when treated with empiric chemotherapy (i.e. paclitaxel and carboplatin or other empiric regimens) versus the alternative hypothesis that it exceeds $60 \%$ with treatment with colorectal site-specific chemotherapy regimens. This design required at least 17 response-evaluable patients with a colorectal molecular signature (who received at least two cycles of chemotherapy), assuming a type I error rate of 5\% and a power of $80 \%$ to reject the null hypothesis when the true objective response rate is equal or exceeds $60 \%$.

Median survival was evaluated by the one arm nonparametric method. The one-sided design tested the null hypothesis that the true median survival is 9 months (range 7 - 11 months) for CUP patients treated with empiric chemotherapy versus the alternative hypothesis that it is equal to or exceeds 20 months (range 18 - 22 months) with treatment with colorectal site-specific regimens. This design required a sample size of 23 patients, assuming a type I error of 5\% and a power of $80 \%$ to reject the null hypothesis when the true median survival is 20 months or longer.

Response to treatment was measured in each patient (RECIST) after at least 2 cycles of chemotherapy, and response rates were calculated. Overall survival was calculated from treatment initiation to death or last follow-up, and survival estimates were made by the Kaplan-Maier method.

\section{Results}

\subsection{Assay Data and Patient Characteristics}

The molecular tumor profile assays were successfully performed in 185 of 213 biopsies (87\%). In 28 specimens $(13 \%)$, the assays could not be performed because of insufficient biopsy material (low RNA yield or RNA preservation). Thirty-two tumors (17\%) had genetic signatures most consistent with colorectal adenocarcinoma (11 of 42 in Cohort A; 21 of 171 in Cohort B).

The clinicopathologic characteristics, therapy received, response to therapy and survival of the 32 patients with a colorectal molecular profile diagnosis are included in Table 1 Cohort A are represented by the first 11 patients, and Cohort B the last 21 patients. There were 18 females and 14 males with a median age of 61 (range $46-81$ ). Twenty-nine of 32 patients $(90 \%)$ had a histologic diagnosis of adenocarcinoma; the majority of patients had two or more sites of metastasis. The pattern of metastasis was typical (liver, peritoneal, omental, pelvic, mesenteric and/or retroperitoneal) of colorectal carcinoma in 29 patients $(90 \%)$. In 30 patients colonoscopy showed no evidence of a primary tumor site; colonoscopy was not done in 2 patients.

\subsection{IHC Evaluation}

All 32 patients had IHC evaluation of their biopsy specimens (Table 1). In 17 of 32 (53\%) patients a specific diagnosis of colorectal carcinoma was highly suggested (CK20+, $\mathrm{CDX} 2+)$. The classic pattern highly supportive of colorectal carcinoma (CK7-, CK20+, CDX2+) was seen in 13 biopsies (40\%), but CK20 (usually present but not specific for colorectal cancer) was positive in 26 tumors (81\%) and CDX2 (usually present in gastrointestinal cancers, particularly in colorectal cancer) was positive in 20 of 26 specimens tested (77\%) Only 3 specimens tested were negative for both CK20 and CDX2 (9\%), but positive for CK7. A total of 12 tumors (37\%) were CK7 positive, a marker not felt to be present in most colon cancers $[16,17]$. The pattern of CK7+, CK20+, CDX2+ as seen in 4 specimens is an immunophenotype described more in rectal carcinomas [18], but could also be present in other upper gastrointestinal adenocarcinomas (e.g. gastric, gastroesophageal, pancreas and biliary tract).

\subsection{Therapy, Response and Survival}

Although this study was not designed to prospectively treat these CUP patients based solely on the molecular assay results, 29 of these 32 patients $(90 \%)$ received first-line (23 patients) and/or second-line (13 patients) chemotherapy known to have activity in advanced colorectal carcinoma (Table 1).

Nineteen patients received first-line colorectal chemotherapy regimens with Folfox or Folfiri (or variants) usually with bevacizumab (see Table 1). Four other patients received first-line chemotherapy with at least one active drug for colorectal cancer. Eight patients received em- 
Table 1. CUP with colorectal molecular profile diagnoses: clinicopathologic characteristics, response to chemotherapy in first and second-line settings and survival.

\begin{tabular}{|c|c|c|c|c|c|c|c|c|c|c|}
\hline \multirow[b]{2}{*}{ \# } & \multirow{2}{*}{ Age } & \multirow{2}{*}{ Sex } & \multirow{2}{*}{ Histology } & \multirow{2}{*}{$\begin{array}{l}\text { Sites of } \\
\text { Metastases }\end{array}$} & \multirow{2}{*}{$\begin{array}{l}\text { IHC + Marker } \\
\text { Stain(s) }\end{array}$} & \multirow{2}{*}{$\begin{array}{l}\text { First-line } \\
\text { Treatment }\end{array}$} & \multicolumn{3}{|c|}{ Second-Line } & \multirow{2}{*}{$\begin{array}{r}\text { Survival } \\
- \text { (Months) }\end{array}$} \\
\hline & & & & & & & Response & Treatment & Response & \\
\hline 1 & 75 & $\mathrm{M}$ & PDA & Liver, lung, bone & CK7 & Folfox & PR & Folfiri/Ce & PR & 19 \\
\hline 2 & 56 & $\mathrm{~F}$ & Adeno & Peritoneum, ovary & $\mathrm{CK} 20, \mathrm{CDX} 2$ & Folfox/B & PR & & & $45+$ \\
\hline 3 & 46 & $\mathrm{~F}$ & PDA & Ovary & $\mathrm{CK} 20, \mathrm{CDX} 2$ & Folfox/B & $\mathrm{CR}$ & & & $64+$ \\
\hline 4 & 61 & $\mathrm{~F}$ & Adeno & Lung, nodes & CK20, villin & $\mathrm{PC}$ & $\mathrm{PD}$ & Cape/RT & PR & 8 \\
\hline 5 & 42 & $\mathrm{M}$ & PDA & Peritoneum, testes & $\mathrm{CK} 20, \mathrm{CDX} 2$ & Folfox & PR & $\mathrm{Iri} / \mathrm{Ce}$ & PR & 38 \\
\hline 6 & 54 & $\mathrm{~F}$ & PDA & Peritoneum, ovary, bone & $\mathrm{CK} 7, \mathrm{CK} 20, \mathrm{CDX} 2$ & $5 \mathrm{FU}$ & NA & $\mathrm{D} / \mathrm{B}$ & $\mathrm{SD}$ & 30 \\
\hline 7 & 53 & $\mathrm{M}$ & Adeno & Liver, peritoneum & CK7, CK20, CEA & $\mathrm{PC}$ & PR & & & 21 \\
\hline 8 & 52 & $\mathrm{~F}$ & PDA & Peritoneum, abdominal Nodes & $\begin{array}{l}\text { CK20, chromogranin, } \\
\text { CK } 7\end{array}$ & No treatment & NA & & & 6 \\
\hline 9 & 70 & $\mathrm{~F}$ & Adeno & Liver & CK7, CK20, CEA & $\mathrm{Gem} / \mathrm{C}$ & $\mathrm{SD}$ & Folfox & PR & $10+$ \\
\hline 10 & 55 & $\mathrm{M}$ & PDA & Liver, bone & CK20, CDX2, CEA & Folfox/B & PR & Iri/Ce & NA & 18 \\
\hline 12 & 61 & M & Adeno & Mesentery, omentum & CK7, CK20 & Folfox/B & PR & & & 12 \\
\hline 13 & 47 & $\mathrm{~F}$ & Adeno & Neck node, lung, Retroperitoneum & $\mathrm{CK} 20$ & Folfox/B & PR & & & $13+$ \\
\hline 14 & 53 & $\mathrm{~F}$ & Adeno & Retroperitoneum, liver & CK7, CK20, CDX2 & Capox/B & PR & & & 6 \\
\hline 15 & 78 & M & $\mathrm{PDC}$ & Pelvic mass & $\mathrm{CK} 20, \mathrm{CDX} 2$ & Folfox/B & PR & & & $33+$ \\
\hline 16 & 53 & M & PDA & Liver, lung, brain & CK7, villin & Capox & PD & & & 17 \\
\hline 17 & 48 & $\mathrm{~F}$ & PDA & Liver, retroperitoneum, Mediastinum & CK7, Her 2-neu & $\mathrm{PC} / \mathrm{T}$ & PR & Capox & $\mathrm{PD}$ & 3 \\
\hline 18 & 63 & $\mathrm{~F}$ & Adeno & Pelvic mass & $\mathrm{CK} 20, \mathrm{CDX} 2$ & Folfox/B & PR & Cape/C/RT & $\mathrm{CR}$ & $68+$ \\
\hline 19 & 63 & $\mathrm{M}$ & Adeno & Peritoneal, ascites & $\mathrm{CK} 7, \mathrm{CK} 20, \mathrm{CDX} 2$ & Capox & PR & & & $15+$ \\
\hline 20 & 54 & $\mathrm{~F}$ & Adeno & Omentum, peritoneal & $\mathrm{CK} 20, \mathrm{CDX} 2$ & $\mathrm{CE}$ & PR & Folfox/B & PR & 60 \\
\hline 21 & 68 & $\mathrm{~F}$ & PDC & Peritoneum, retroperitoneum & CK20 & $\mathrm{PC}$ & PR & & & $5+$ \\
\hline 22 & 46 & $\mathrm{M}$ & PDC & Mediastium & CK20, CDX2 & Folfox/Iri/B & PR & & & $44+$ \\
\hline 23 & 81 & $\mathrm{~F}$ & Adeno & Liver, lung & $\mathrm{CK} 20, \mathrm{CDX} 2$ & Folfox/B & PR & & & $10+$ \\
\hline 24 & 49 & $\mathrm{~F}$ & Adeno & Liver, peritoneum & $\mathrm{CDX} 2$ & PCE & PR & Gem/Iri & PD & 21 \\
\hline 27 & 69 & M & Adeno & Pelvic mass, bone & $\mathrm{CK} 20, \mathrm{CDX} 2$ & Cape/RT & $\mathrm{CR}$ & & & $38+$ \\
\hline 28 & 64 & $\mathrm{~F}$ & Adeno & Ovary, omentum & $\mathrm{CK} 20, \mathrm{CDX} 2$ & Folfox/B & CR & Folfiri & $\mathrm{PD}$ & 21 \\
\hline 29 & 51 & $\mathrm{M}$ & Adeno & Retroperitoneum & CK20 & Capox & NA & & & $17+$ \\
\hline 30 & 49 & M & PDA & Liver & $\mathrm{CK} 20, \mathrm{CDX} 2$ & Iri/Gem & $\mathrm{SD}$ & & & 10 \\
\hline 31 & 47 & $\mathrm{~F}$ & PDA & Liver, lung, mesentery & $\mathrm{CK} 20, \mathrm{CDX} 2$ & Cape/Iri/B & PR & & & 20 \\
\hline 32 & 62 & $\mathrm{M}$ & Adeno & Retroperitoneal mass & CK7, CK20 & $5 \mathrm{FU} / \mathrm{P} / \mathrm{C}$ & $\mathrm{PD}$ & & & 5 \\
\hline
\end{tabular}

IHC, immunohistochemistry; PDA, poorly differentiated adenocarcinoma; PDC poorly differentiated carcinoma; Adeno, adenocarcinoma; Gem, gemcitabine; Folfox, fluorouracil/leucovorin/oxaliplatin; Folfiri, fluorouracil/leucovorin/irinotecan; Iri, irinotecan; 5FU, fluorouacil; D, docetaxel; B, bevacizumab; Cis, cisplatin; Cape, capecitabine; Capox, capecitabine/oxilaplatin; P, paclitaxel; C, carboplatin, E, etoposide; T, trastuzumab; RT, radiotherapy; Ce, cetuximab; M. male, F, female; PR, partial response; CR, complete response; SD, stable disease; PD, progressive disease; NA, not accessible; CUP, cancer of unknown primary site.

piric first-line chemotherapy, usually on clinical trials, and 6 of these 8 patients received second-line colorectal regimens. Seven patients who received first-line chemotherapy with colorectal regimens received alternative colorectal regimens as second-line chemotherapy. Nine patients received second-line chemotherapy with colorectal regimens with Folfox or Folfiri (or variants). Four additional patients received second-line chemotherapy with at least one active drug for colorectal cancer. One patient received no therapy.

Seventeen of 23 patients (74\%) who received first-line colorectal chemotherapy regimens had an objective re- sponse to chemotherapy and 7 of 13 (54\%) who received second-line colorectal chemotherapy regimens had objective responses (Table 1). Objective response rates to first-line and second-line chemotherapy for all patients were $69 \%$ (22 of 32) and 54\% (7 of 13) respectively.

The median, 2 year and 4 year survivals of all 32 patients were 21 months, $(95 \%$ confidence intervals $=17.1$ - 24.9 months) $42 \%$ and 35\% respectively (Figure 1). The median survival of 29 patients receiving colorectal chemotherapy regimens as first-line and/or second-line therapy was 22 months (Figure 2). The median survival of the 23 patients receiving first-line colorectal regimens 


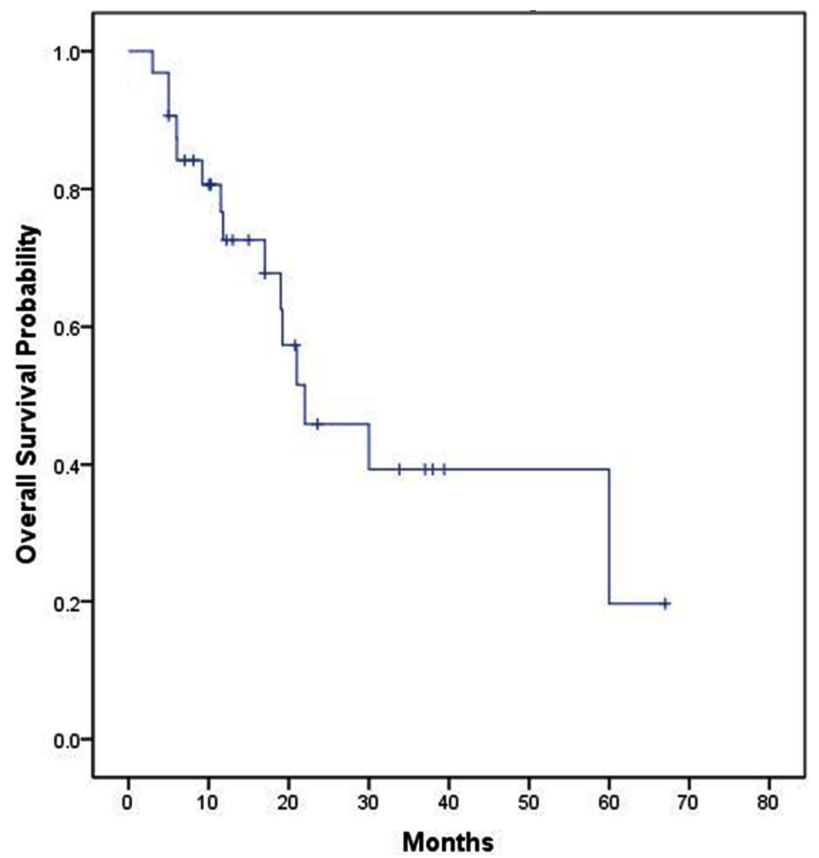

Figure 1. Overall survival of all 32 patients.

was 22 months (Figure 3).

\section{Discussion}

Chemotherapy for the majority of the patients with CUP has been with empiric regimens in the past, and relatively ineffective for most patients (median survival about 9 months) [12]. The major difficulties have been lack of knowledge of the primary site of origin and ineffective treatment for most patients with advanced solid tumors. Broad spectrum empiric chemotherapy regimens were developed in the past 15 years in hopes of treating some of the more responsive tumor types present in this diverse clinicopathologic syndrome. The empiric regimens have activity in several tumor types known to be occult primary sites in some CUP patients (e.g. lung, ovary, breast). Others, such as colorectal carcinoma are not effectively treated by most of the empiric regimens for CUP. This lack of activity against colorectal cancer was previously unimportant, since no effective treatment existed for these patients. In recent years, the use of relatively site-specific colorectal chemotherapy regimens has improved the prognosis for patients with advanced colorectal carcinoma patients, with median survivals now approaching 24 months $[13,14]$. Therefore the recognition or diagnosis of the subset of CUP patients presenting with a clinically undetectable primary colorectal adenocarcinoma is potentially important.

Improved IHC staining and the introduction of gene expression profiling are diagnostic techniques that are likely to be of value in identifying this subset of "colo-

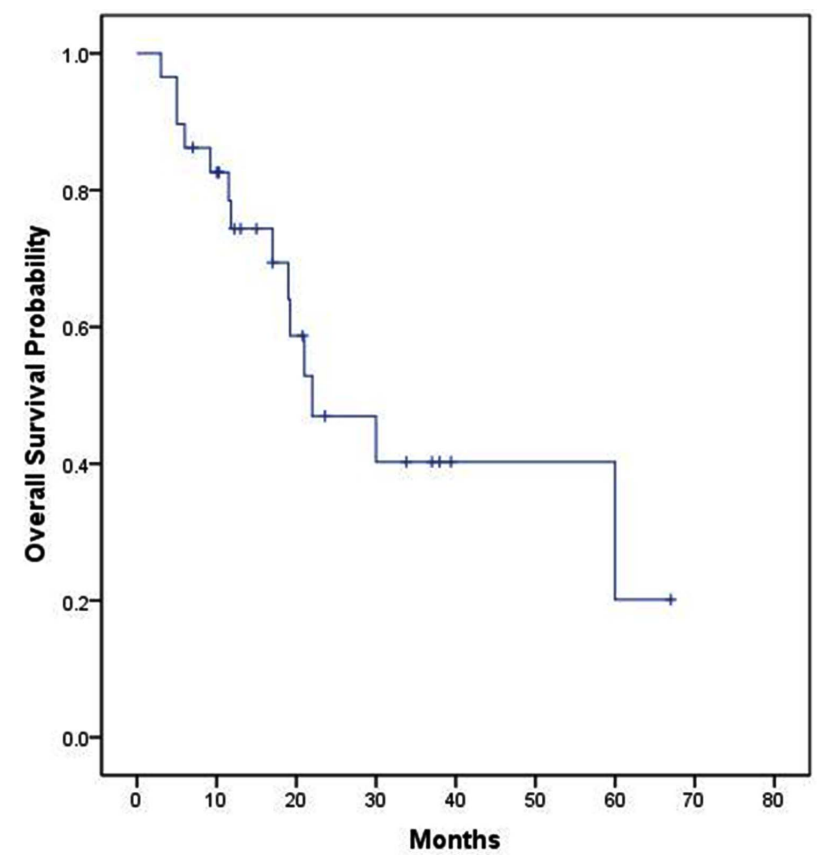

Figure 2. Overall survival of patients receiving first- and/or second-line colorectal chemotherapy regimens $(n=29)$.

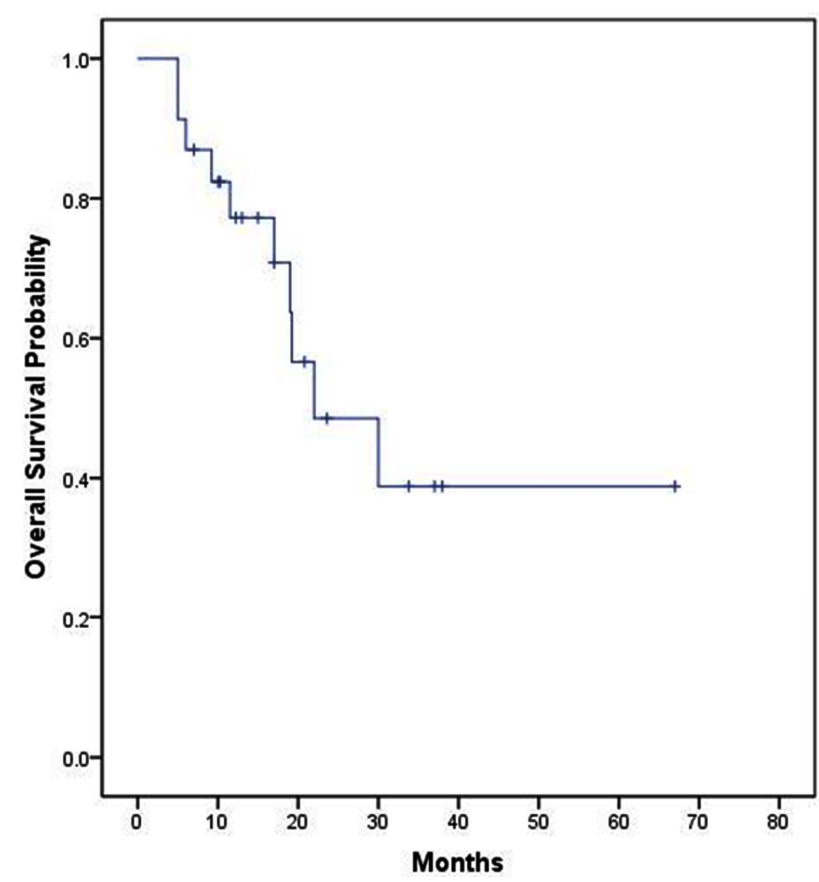

Figure 3. Overall survival of patients receiving first-line colorectal chemotherapy $(n=23)$.

rectal profile" CUP patients. In this prospective evaluation of biopsy specimens from patients at two institutions, 213 biopsies from CUP patients were tested by two different molecular profile assays, and 32 were given a presumptive diagnosis of colorectal carcinoma. In the majority of these patients, subsequent treatment decisions in 
the first and second-line settings were based on the clinicopathologic features and the molecular assay diagnosis.

The clinical and pathologic features of these 32 CUP patients were consistent with a diagnosis of advanced colorectal carcinoma. This high correlation of standard clinicopathologic features also supports the accuracy of the molecular assay diagnoses. The sites of metastasis were fairly typical in $29(90 \%)$ patients, and the IHC staining patterns of the biopsy specimens were frequently supportive of colorectal carcinoma. The IHC staining patterns are also helpful in recognizing this subset of CUP patients and typical IHC staining patterns may be useful in planning therapy, as has been previously reported by Varadhachary et al. [19] A minority of the patients reported here did not have typical IHC staining patterns for colorectal carcinoma, and the molecular assays provided a presumptive diagnosis in these patients.

Twenty-nine of these 32 patients received first-line and/or second-line chemotherapy regimens known to be relatively effective in advanced colorectal carcinoma. The response rate to first-line colorectal chemotherapy regimens was $74 \%$ ( 17 of 23 ); median survival was 22 months, with 2 year and 4 year survivals of $48 \%$ and $35 \%$, respectively (Figure 2).

This study was not specifically designed as a prospective evaluation of therapy based on the molecular assay diagnosis, but rather a prospective study of biopsy specimens to correlate the molecular assay results with clinicopathologic features. The chemotherapy administered was not the same for all patients.

However, the majority of patients (29 of 32) received either first-line and/or second-line chemotherapy with regimens known to useful for patients with advanced colorectal cancer. Second-line chemotherapy with colorectal regimens was useful in more than one-half of the patients treated, further adding to the overall survival in these patients.

The median, 2 year and 4 year survival data $(21$ months, $42 \%$ and $35 \%$ respectively) for all 32 patients with CUP diagnosed as colorectal by molecular assay is very similar to patients with known metastatic colorectal carcinoma, and superior to the expected survival of CUP patients who receive empiric chemotherapy (median survival 9 months). These data support the concept of considering colorectal site-specific chemotherapy for CUP patients who have molecular profile assay and/or IHC diagnoses of colorectal carcinoma. These patients are a newly recognized favorable subset of CUP (colorectal profile-CUP), and are candidates for colorectal site-specific chemotherapy. It is unclear now if the KRAS and BRAF status of these tumors match that of known advanced colorectal cancer, or if the use of biologics including cetuximab, in KRAS wild type tumors will prove to be beneficial. Additional prospective biologic studies and site-specific chemotherapy directed by molecular assay diagnosis in CUP are warranted.

\section{REFERENCES}

[1] F. A. Greco amd J. D. Hainsworth, "Cancer of Unknown Primary Site," In: V. T. J. DeVita, S. Hellman and S. A. Rosenberg, Eds., Cancer: Principles and Practice of Oncology, 8th Editon, Lippincott, Philadelphia, 2008, pp. 2363-2387.

[2] F. A. Greco and M. G Erlander, "Molecular Classification of Cancers of Unknown Primary Site," Molecular Diagnosis Therapy, Vol. 13, No. 6, 2009, pp. 367-737.

[3] X. J. Ma, R. Patel, X. Wang, et al., "Molecular Classification of Human Cancers Using a 92-Gene Real-Time Quantitative Polymerase Chain Reaction Assay," Archives of Pathology \& Laboratory Medicine, Vol. 130, No. 4, 2006, pp. 465-472.

[4] N. Rosenfeld, R. Aharonov, E. Meiril, et al., "MicroRNAs Accurately Identify Cancer Tissue Origin," Nature Biotechnology, Vol. 26, No. 4, 2008, pp. 462-469. doi:10.1038/nbt1392

[5] F. A. Monzon, M. Lyons-Weiler, L. J. Buturovic, et al., "Multicenter Validation of a 1500-Gene Expression Profile for Identification of Tumor Tissue of Origin," Journal of Clinical Oncology, Vol. 27, No. 15, 2009, pp. 25032509. doi:10.1200/JCO.2008.17.9762

[6] R. Pillai, R. Deeler, C. T. Rigl, et al., "Validation and Reproducibility of a Microarray-Based Gene Expression Test for Tumor Indentification in Formalin-Fixed, Paraffin-Embedded Specimens," The Journal of Molecular Diagnostics, Vol. 13, No. 1, 2011, pp. 48-56. doi:10.1016/i.jmoldx.2010.11.001

[7] G. Varadhachary, D. Talantov, M. Raber, et al., "Molecular Profiling of Carcinoma of Unknown Primary and Correlation with Clinical Evaluation," Journal of Clinical Oncology, Vol. 26, No. 27, 2008, pp. 4442-4450.

doi:10.1200/JCO.2007.14.4378

[8] J. Bridgewater, R. van Laar, A. Floore, et al., "Gene Expression Profiling May Improve Diagnosis in Patients with Carcinoma of Unknown Primary," British Journal of Cancer, Vol. 98, No. 8, 2008, pp. 1425-1430. doi:10.1038/sj.bjc. 6604315

[9] H. M. Horlings, R. van Laar, J.-M. Kerst, et al., "Gene Expression Profiling to Identify the Histogenetic Origin of Metastatic Adenocarcinomas of Unknown Primary," Journal of Clinical Oncology, Vol. 26, No. 27, 2008, pp. 4435-4439. doi:10.1200/JCO.2007.14.6969

[10] F. A. Monzon, F. Medeiros, M. Lyons-Weiler, et al., "Identification of Tissue of Origin in Carcinoma of Unknown Primary with a Microarray-Based Gene Expression Test," Diagnostic Pathology, Vol. 5, 2010, pp. 3-7. doi:10.1186/1746-1596-5-3

[11] F. A. Greco, D. R. Spigel, D. A. Yardley, et al., "Molecular Profiling in Unknown Primary Cancer Tissue of Origin Prediction," The Oncologist, Vol. 15, No. 5, 2010, 
pp. 500-506. doi:10.1634/theoncologist.2009-0328

[12] F. A. Greco and N. Pavlidis, "Treatment for Patients with Unknown Primary Carcinoma and Unfavorable Prognostic Factors," Seminars in Oncology, Vol. 36, No. 1, 2009, pp. 35-74. doi:10.1053/j.seminoncol.2008.10.005

[13] L. B. Saltz, S. Clarke, E. Diaz-Rubio, W. Scheithauer, et al., "Bevacizumab in Combination with Oxaliplatin-Based Chemotherapy as First-Line Therapy in Metastatic Colorectal cancer: A Randomized Phase III Study," Journal of Clinical Oncology, Vol. 26, No. 12, 2008, pp. 2013-2019. doi:10.1200/JCO.2007.14.9930

[14] F. F. Kabbinavar, H. I. Hurwitz, J. Yi, et al., "Addition of Bevacizumab to Fluorouracil-Based First-Line Treatment of Metastatic Colorectal Cancer: Pooled Analysis of Cohorts of Older Patients from Two Randomized Clinical Trials," Journal of Clinical Oncology, Vol. 27, No. 2, 2000, pp. 199-205. doi:10.1200/JCO.2008.17.7931

[15] D. Talantov, J. Baden and T. Jaktoe, "A Quantitative Reverse Transcriptase-Polymerase Chain Reaction Array to Identify Metastatic Carcinoma Tissue of Origin," The
Journal of Molecular Diagnostics, Vol. 8, No. 3, 2006, pp. 320. doi:10.2353/jmoldx.2006.050136

[16] S. Y. Park, B. H. Kim, S. H. Kim, et al., "Panels of Immunohistochemical Markers Help Determine Primary Sites of Metastatic Adenocarcinomas," Archives of Pathology \& Laboratory Medicine, Vol. 131, No. 10, 2007, pp. 15611567.

[17] K. A. Oien, "Pathologic Evaluation of Unknown Primary Cancer," Seminars in Oncology, Vol. 36, No. 1, 2009, pp. 8-37. doi:10.1053/j.seminoncol.2008.10.009

[18] P. J. Zhang, M. hah, G. W. Spiegel, et al., "Cytokeratin 7 Immunoreactivity in Rectal Adenocarcinoma," Applied Immunohistochemistry \& Molecular Morphology, Vol. 11, No. 4, 2003, pp. 306-310. doi:10.1097/00129039-200312000-00005

[19] G. R. Varadhachary, M. N. Raber, A. Matamoros, et al., "Carcinoma of Unknown Primary with a Colon-Cancer Profile-Changing Paradigm and Emerging Definitions," The Lancet Oncology, Vol. 9, No. 6, 2008, pp. 596-600. doi:10.1016/S1470-2045(08)70151-7 